\title{
SHEAR STRESS MEASUREMENTS AT THE SEA BOTTOM BY MEANS OF FERROFLUIDS
}

\author{
A. Viviano ${ }^{1}$, L.M. Stancanelli ${ }^{1}$, R.E. Musumeci ${ }^{1}$, E. Foti ${ }^{1}$
}

\begin{abstract}
An innovative measurement technique is proposed for investigating shear stress at sandy bottoms. This technique is based on the adoption of a ferrofluidic sensor and of an optical readout strategy. An experimental campaign is carried out for evaluating its performance. Experiments differ for the ferrofluidic sensor configuration (difference in the magnetic field) and for the bottom configurations (fixed bed or sandy bed). Calibration of the ferrofluidic sensor for the range of the investigated hydraulic condition and of the controlling magnetic field is presented. The ferrofluidic technique is promising when applied at sandy bottoms, as neither adhesion processes between sand grains and ferrofluid or influence of impacts of grains on the measurement are observed. In particular, the preliminary measure performed indicated that the ferrofluidic sensor is capable of sensing the different bed roughness.
\end{abstract}

Keywords: magneto-rheological fluid; bottom shear; laboratory instrument

\section{INTRODUCTION}

Flow-induced shear stresses control sediment transport in coastal areas. This is important in highly dynamic dissipative boundary conditions such as in the swash zone (Lo Re et al., 2012; Viviano et al., 2015) or in complex wave-current interaction processes over rough beds (Faraci et al., 2008; Lim and Madsen, 2016) or else in the presence of gravity currents propagation in the coastal areas (Musumeci et al., 2018; Stancanelli et al., 2018). Unfortunately, the direct measurement of shear stresses at the sea bottom is a complex task, particularly in the presence of sediments, where state-of-art instruments, as hot film, may be heavily damaged (Wallace and Vukoslavčević, 2010).

To overcome such limits, an innovative methodology is applied here, based on ferrofluids. These are colloidal suspensions made up by nanoparticles of ferromagnetic materials dispersed in a non-magnetic solvent, with magneto-rheological characteristics. An imposed magnetic field causes the deformation of ferrofluids, which return to their initial condition once the magnetic field is removed.

Such a phenomenon, called Rosensweig effect, was exploited recently to measure bottom shear stresses by means of an inductive readout strategy (Musumeci et al., 2015a,b, 2018). A ferrofluidic sensor is generated, placing a tiny drop of ferrofluid excited by a magnetic field onto two planar coils. The latter system is capable to sense the movements of the ferrofluid sensor, and to transduce them into an output voltage. Results of such inductive readout strategy were quite good, also considering that the robustness of the technique was also evaluated for different hydraulic regimes, i.e. steady, unsteady and oscillatory flow. However, in all cases low-sensitivity region of the ferrofluidic sensor was observed for lower values of shear stresses.

The aim of the present research is to evaluate the possibility to measure shear stress using optical observations of the ferrofluid deformations and, movements induced by the flow. The advantage to adopt an optical system is the capability to gain information about the ferrofluid response (displacement, drop separation etc.) and about the sediment transport when in the presence of mobile bed.

The remainder of this paper is organized as follows. A first section describes the research methods, including information about the proposed measurement technique, the experimental apparatus and procedure adopted. The following section reports the hydraulic parameters characterizing the different tests as well as about the bottom configurations adopted. Then we present the experimental results about the measurement performances in case of different ferrofluidic sensor configurations and different bottom configurations. Finally, a final section reports the conclusions.

\section{METHODS}

\section{Ferrofluids and the optical readout strategy}

Ferrofluids are here adopted as shear stress measurement sensor, taking advantage of the peculiar characteristic of this type of fluids. The main characteristic of these fluids is their deformability in the presence of a permanent magnetic field (Odenbach, 2002; De Vicente et al., 2011). Figure 1a shows a typical example of ferrofluid surface deformation in the presence of an applied permanent magnetic field. Multiple spikes at the fluid surface interface, aligned along the direction of the magnetic streamlines, form as a consequence of the phenomenon referred to as Rosensweig effect (Cowley and Rosensweig, 1967). Such phenomenon is here exploited to measure bottom shear stresses by means of an optical readout strategy.

\footnotetext{
${ }^{1}$ Department of Civil Engineering and Architecture, University of Catania, Italy
} 
Principle of this measurement technique is to evaluate the optical deformation of a ferrofluid spike induced by the water-flow impacting on it. A sketch of the principle of operation in still water and in the presence of a current is presented in Figure 1b,c. For still water conditions the ferrofluid drop assumes a stable symmetrical conical shape. A deformation of the spike is observed in the case of the dynamic condition. In particular, the ferrofluid assumes a shape more elongated along the flow direction. The idea here to correlate the deformation and movement of the ferrofluid spike to the bottom shear stresses of the external flow.

a)

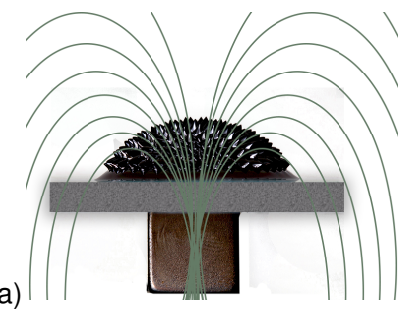

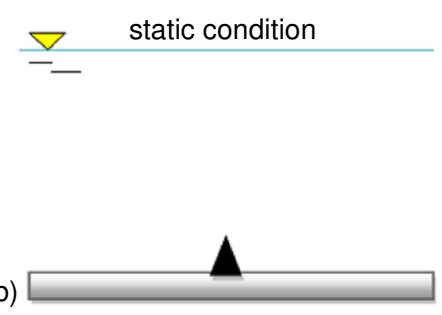

c)

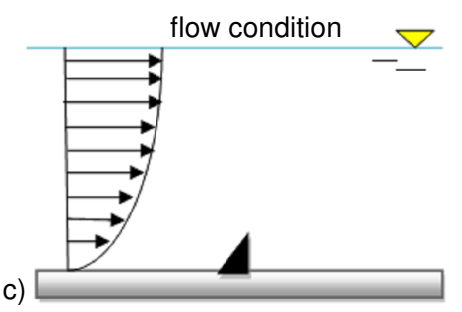

Figure 1: a) Scheme of the Rosensweig effect with the ferrofluid deformation induced by the action of a permanent magnet. b) Ferrofluid drop in static conditions; c) Ferrofluid drop deformed in the presence of a flow.

\section{The experimental apparatus and procedure}

The performances of the proposed technique have been evaluated in steady current conditions. The experimental flume is $3.65 \mathrm{~m}$ long, $0.15 \mathrm{~m}$ wide, $0.42 \mathrm{~m}$ high. The experimental apparatus is made up by two reservoirs and a recirculation system, that is controlled by a pump and a valve. The latter allow to control the flow discharge with a great accuracy. For independently evaluating the hydrodynamics inside the channel, we adopted a Vectrino profiler. This is located in the same section of the ferrofluidic sensor, about $2.35 \mathrm{~m}$ from the inlet. Figure 2 shows the experimental flume, where the Vectrino Profiler, the ferrofluid sensor, and the optical systems are located at the same measuring section. Here a known permanent magnetic field is applied to produce a ferrofluid spike, using a drop of ferrofluid, whose volume is about $10 \mu 1$, located over a plastic disk of height $7 \mathrm{~mm}$ (view close-up view in Figure 2). The bottom channel elevation is the same than that of the ferrofluid drop base, both for the rigid or sandy bottom cases.

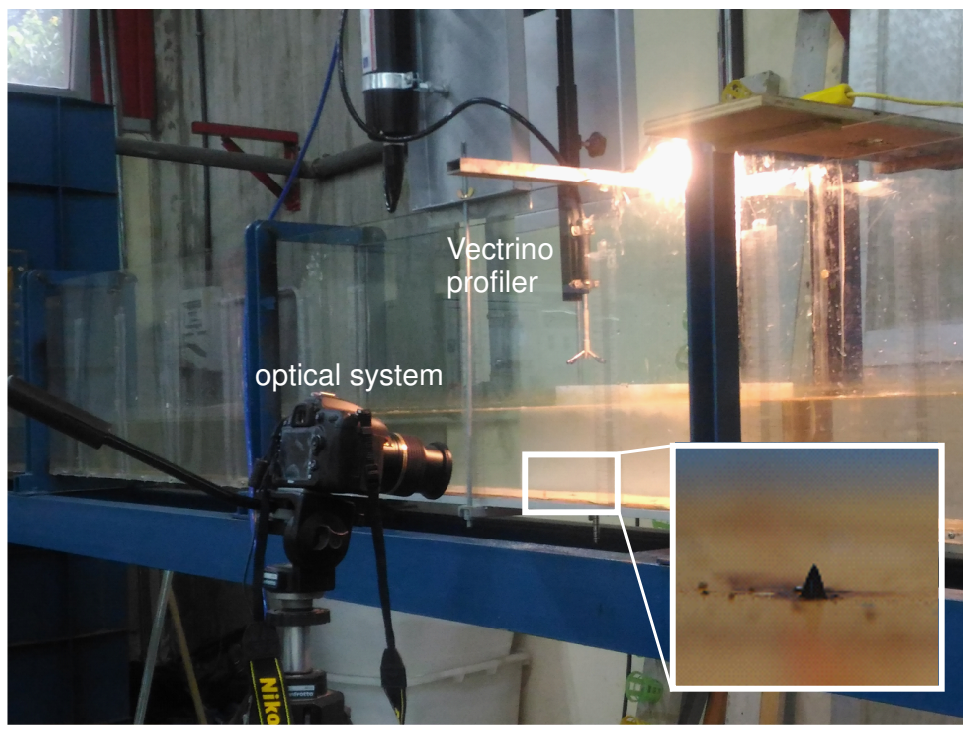

Figure 2: Experimental apparatus consisting in an hydraulic channel where two measurement instruments are adopted the ferrofluid sensor at the channel bottom and a Vectrino profiler. 
For evaluating the shape deformation of the ferrofluidic sensor, as well as the sediment status (no motion, incipient motion, sediment transport), a camera is located in front of the wall. A Nikon camera D7200 mounting a fixed focus length of $90 \mathrm{~mm}$ is used to this aim.

A wide range of the deformation descriptors could be used, for example: apex displacement, centroid displacement, drop section area and so on. In the presence of mobile beds the one which is more robust to optically describe changes in the ferrofluidic sensor results to be the apex displacement, since its evaluation is not disturbed by the presence of sand movements. In the following, the measure of the ferrofluidic sensor deformation related to the shear stress is reported always considering the apex displacement parameter.

The experimental procedure for performing an experimental cycle is as follows. The preliminary operation consists of preparing the channel bed, locating a rigid smooth bottom or a mixture of sand. The rigid bottom is a rigid plastic layer with thickness of $7 \mathrm{~mm}$, whereas in the case of movable sandy bottom a sandy layer of the same thickness is placed at the channel bottom. In the latter case the sandy bottom is flattened using an appropriately device.

After that, the ferrofluid sensor is generated by locating a set of permanent magnets under the channel bed and a drop of ferrofluid above the channel bed. The channel is filled with water until the desired water level is reached. Then the setting of the circulation system useful for the development of hydraulic regime is carried out. In the present experimental campaign we consider steady currents. More information about flow parameters are given in the next section. The optical system is set up by fixing its optical parameters. The camera is switched-on and maneuvered by using a remote control device. This allows to minimize any movement of the camera. The videorecord of the ferrofluid deformation in the static condition is acquired for $240 \mathrm{~s}$ at $50 \mathrm{fps}$. Then the camera is switched-off and a hydraulic steady current is established. Sufficient time for hydraulic regime stabilization is given before starting a new video acquisition. This sequence is repeated for each test condition. When all the hydraulic regimes are reproduced the test cycle is concluded. Finally, keeping the camera at the same position, some pictures of a meter are acquired, which are useful for post processing calibration and elaboration of the images. In particular, the latter pictures are used for converting pixel coordinates to real world measure.

\section{EXPERIMENTS}

All experiments are performed with the hydraulic channel having a slope of $1.80 \%$. Each experiment can be considered as a cycle of tests ran adopting the same bottom configuration and exploring different steady current conditions. For each experiment cycle we run 15 tests, investigating a velocity range that varies from 0 to $0.25 \mathrm{~m} / \mathrm{s}$ in the undisturbed layer. The channel bottom configurations examined are rigid bottom and sandy bottom. The latter consist in a bimodal mixture. We adopted two different types of sand: fine sand $\left(d_{50}=0.25 \mathrm{~mm}\right)$ and coarse sand $\left(d_{50}=0.56 \mathrm{~mm}\right)$. For the formation of the ferrofluid drop, we adopted two different types of permanent magnets both of them produced by Supermagnete Inc: the S-0805 (each magnet with a nominal magnetic strength of 19.6 N) and S-08-08 (each magnet with a nominal magnetic strength of $24.5 \mathrm{~N}$ ). Table 1 reports the main characteristics of each test cycle composing the experimental campaign.

Table 1: Controlling parameters of the different experimental cycles. In particular, there are reported: the cycle identification name, the type of bottom, the composition of the bottom layer in term of volume concentration [\%], the number of steady currents tested, the type of magnets used reporting respectively number of magnets and the individual magnetic strength [N].

\begin{tabular}{|c|c|c|c|c|c|}
\hline $\begin{array}{l}\text { cycle } \\
\text { id name }\end{array}$ & $\begin{array}{l}\text { bottom } \\
\text { type }\end{array}$ & $\begin{array}{l}\text { sedimen } \\
\text { fine sanc }\end{array}$ & $\begin{array}{l}\text { me concentration } \\
\text { coarse sand }\end{array}$ & $\begin{array}{c}\text { hydraulic configurations } \\
\text { no. }\end{array}$ & $\begin{array}{c}\text { magnets } \\
\text { no. and magnetic strength }\end{array}$ \\
\hline 1 & rigid & - & - & 13 & $6 \times 19.6 \mathrm{~N}$ \\
\hline 2 & rigid & - & - & 13 & $6 \times 19.6 \mathrm{~N}$ \\
\hline 3 & rigid & - & - & 13 & $6 \times 19.6 \mathrm{~N}$ \\
\hline 4 & rigid & - & - & 13 & $6 \times 24.5 \mathrm{~N}$ \\
\hline 5 & rigid & - & - & 13 & $6 \times 24.5 \mathrm{~N}$ \\
\hline 6 & mixture & $70 \%$ & $30 \%$ & 11 & $6 \times 24.5 \mathrm{~N}$ \\
\hline 7 & mixture & $70 \%$ & $30 \%$ & 11 & $6 \times 24.5 \mathrm{~N}$ \\
\hline
\end{tabular}




\section{RESULTS}

For the development of the proposed measurement technique the relation between the ferrofluidic sensor deformation and the shear stress acting on it must be known. This relation essentially describes the functioning curve of the ferrofluidic sensor when a readout optical approach is adopted.

In order to calibrate the present technique, each hydraulic regime is associated with an independent measure of the time-averaged shear stress at the bottom. Estimate of the mean bottom shear stress is performed evaluating fitting the wall log law to the time-averaged velocities acquired by means of the Vectrino Profiler. Additionally, the velocity acting at the elevation of the ferrofluid spike height (about 1,5 $\mathrm{mm}$ from the bottom) is determined. Since the height of the ferrofluid drop is very small, also the velocity profiles in the viscous sublayer and the buffer layer are considered in the analysis to determine the relative position of the ferrofluid within the boundary layer. For example, in Figure 3 the tip of the ferrofluid is located within the buffer layer. More in depth description about this procedure can be found in previous publication by the authors (Musumeci et al., 2018).

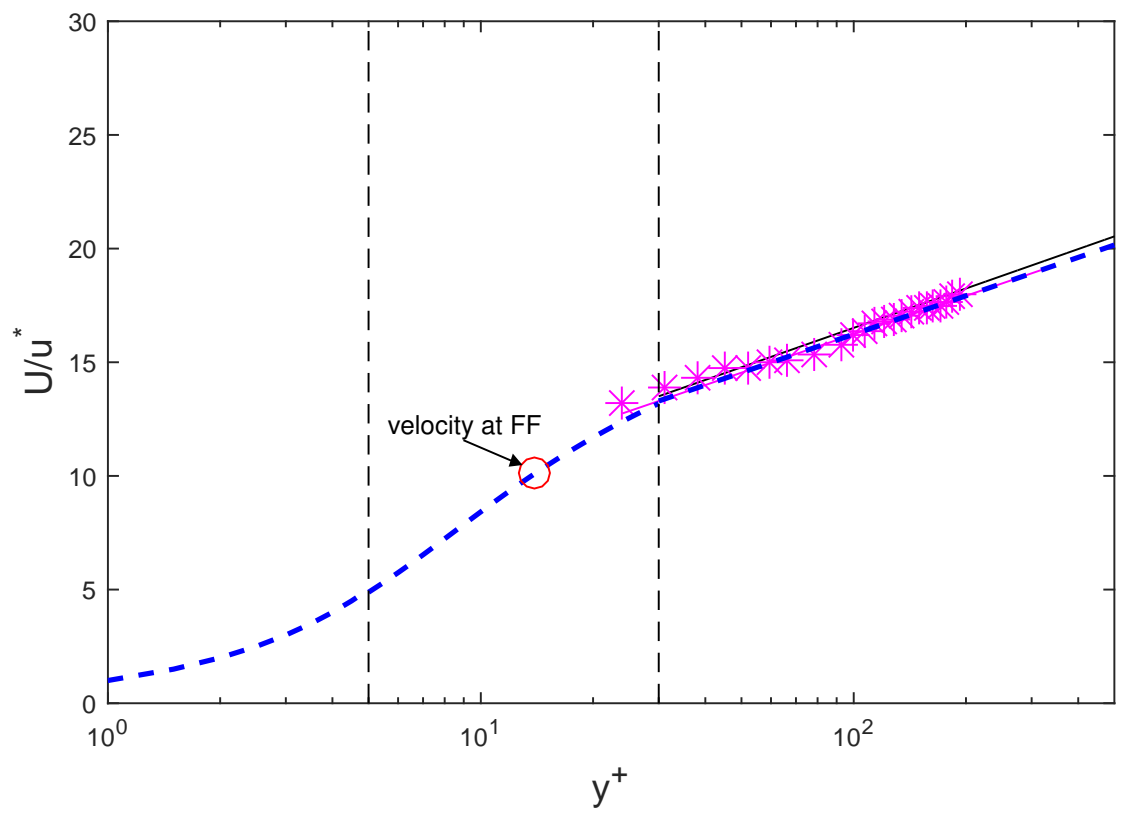

Figure 3: Estimate of bottom shear stress and velocity at the ferrofluid location obtained by applying a best fit procedure of measured time-averaged velocities in the log layer, buffer layer and viscous sub-layer.

Experimental evidences obtained from the present experimental campaign are useful for investigating the capability of the proposed technique to work considering different ferrofluidic sensor and bottom configurations. First of all, we analyze the influence of the ferrofluidic sensor configuration. Here, we present an analysis of experimental cycles ( 1 to 5 in Table 1) carried out over a rigid bed.

For each experiment cycle, the same hydraulic regime range is examined. The relation between observed ferrofluid drop deformation (apex displacement) and shear stress is determined and reported in Figure 4. In particular, in Figure 4a and Figure $4 \mathrm{~b}$ we report experimental observations acquired when the ferrofluidic sensor is characterized by lower magnetic intensity ( 8 magnets with individual nominal strength of $19.6 \mathrm{~N}$ ) and higher magnetic intensity (8 magnets with individual nominal strength of $24.5 \mathrm{~N}$ ), respectively. For the hydraulic regimes analyzed, this relation is always a linear. As expected, at higher shear stress corresponds higher ferrofluid deformation. From the comparison of data coming from repeated experimental cycles, the ferrofluidic sensor shows lower performance in terms of experimental repeatability when a lower intensity of the magnets is used. On the other hand, a higher accuracy of the ferrofluidic sensor is observed for higher magnetic intensity configuration when the optical readout strategy is adopted. Such observation, it is probably due to the fact that in the presence of higher magnetic intensity the ferrofluid drop assumes a more rigid behavior. The rigidity of the ferrofluidic sensor results in a more clear oscillation of its tip when impacted by the water flow and consequently in a more detectable deformation. 

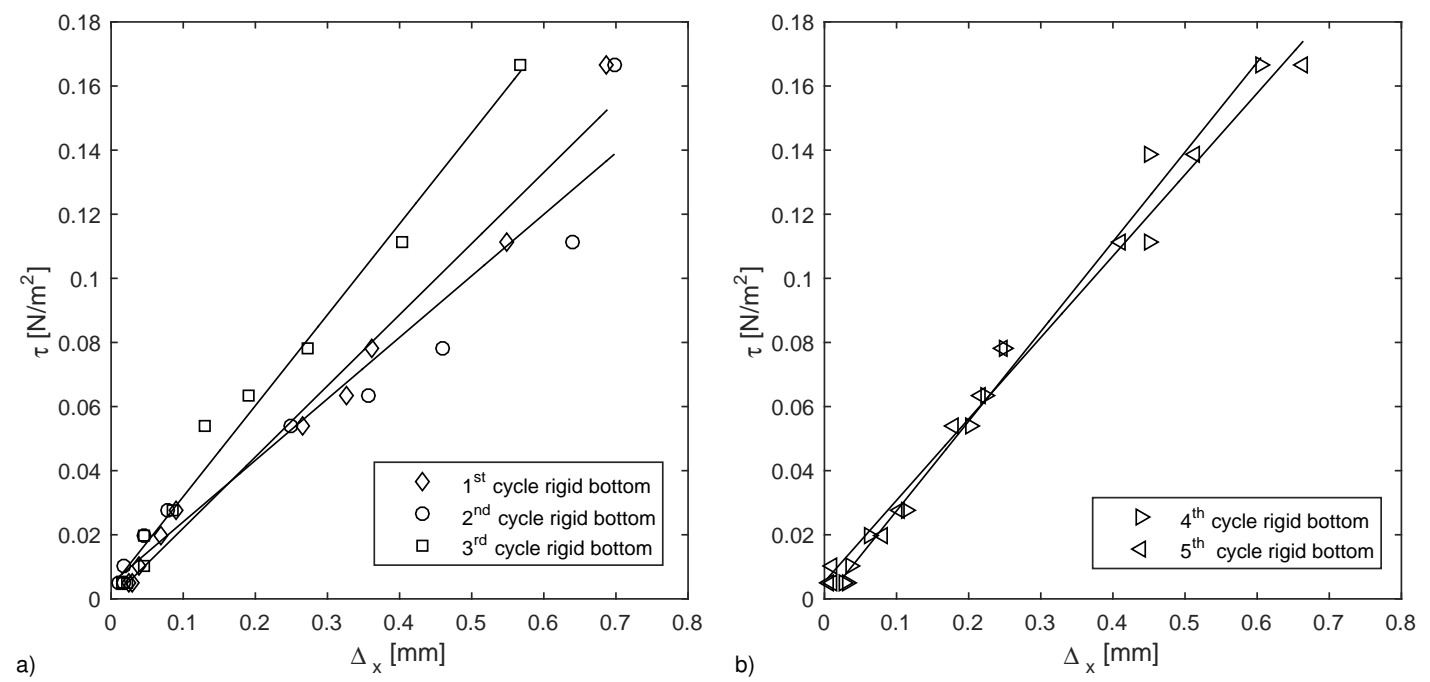

Figure 4: Effect of the magnetic field strength on the relationship between ferrofluid apex displacement and mean bottom shear stress: a) lower magnetic field strength (6 magnets with an individual nominal strength of $19.6 \mathrm{~N}$ ) b) higher magnetic field strength (6 magnets with an individual nominal strength of $24.5 \mathrm{~N})$.

Other analysis on the present experimental evidences regards the capability of the proposed measurement technique to work in the presence of movable bed. Preliminary experiments are here presented carried out for a bottom made up by a bimodal mixture composed by a concentration in volume of $70 \%$ of fine sediment $\left(d_{50}=0.24 \mathrm{~mm}\right)$ and a $30 \%$ of coarse sediment $\left(d_{50}=0.56 \mathrm{~mm}\right)$. Figure 5 shows the behavior of the ferrofluic sensor in the presence of sandy bottom when characterized by higher magnetic configuration. For these experimental cycles we ended the cycle when the incipient motion of sediments is observed. Hence no data above the threshold of motion are shown. Also in the presence of movable bed the ferrofluidic sensor is characterized by a linear relation between ferrofluid deformation and shear stress. Estimates on the functioning relation acquired for rigid bottom (see Figure $4 \mathrm{~b}$ ) are compared with the one presented here over sandy bottom. The range of deformation is comparable with the one over the rigid bottom although in the case of sandy bottom the data are a bit more scattered. This may be caused by the fact that the presence of a bed slope induces a sediment motion triggering that is not simultaneous in time for all sections of the channel. Upstream sand movement and dune formation are responsible for changing the hydrodynamics acting at the channel bottom of downstream section. Such a phenomenon cannot be caught correctly the time-averaged velocity profiles estimates and it seems responsible for the differences in terms of angular coefficient characterizing the different linear trends observed in Figure 5.

\section{CONCLUSION}

An experimental campaign for evaluating the performance of an innovative measurement technique is presented. The technique is based on the use of ferrofluid coupled with optical system for detecting ferrofluid shape deformation under different hydraulic regimes. Cycle of steady current tests are run for evaluating the robustness of the technique in different configurations of the ferrofluidic sensor and of the bottom channel. In all tests performed it is observed a relation between the ferrofluid deformation and the bottom shear stress characterized by a linear trend. The rigid bottom configuration is adopted for evaluating the influence of sensor configuration. The higher test repeatability rate observed for ferrofluidic sensor with higher magnetic field indicates higher sensor stability under this configuration. Indeed, such a configuration is later adopted for all other tests. Preliminary tests with sandy bottom show results that are in agreement with the relation gathered for rigid bottom. Also in these cases linear trends are observed, although the results show lower degree of repeatability when compared with the rigid bottom case. The latter phenomenon is presumably induced by the channel slope, which is responsible of the incipient motion in sections upstream of the measuring one, that evolves in dune formation downstream. Further experimental tests should be run in the future for evaluating the performance over sandy bottoms considering a wider 


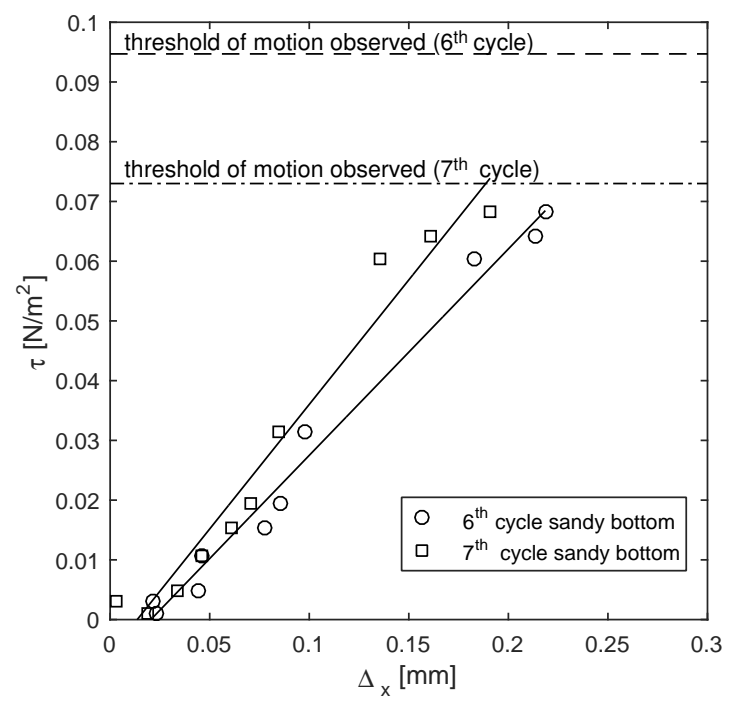

Figure 5: Ferrofluid drop apex displacement vs. mean bottom shear stress for two different test cycles carried out in the presence of a sandy bottom.

range of sediment characteristics, in order to evaluate the sensibility of the measurements.

\section{ACKNOWLEDGEMENTS}

This work has been partly funded by the EU project HYDRALAB PLUS (proposal number 64110), by the project NEWS - Nearshore hazard monitoring and Early Warning System (code C-3.2-60) in the framework of the programme INTERREG V-A Italia Malta 2014-20, and by University of Catania project Interazione onde-correnti nella regione costiera (INOCS).

\section{References}

M. D. Cowley and R. E. Rosensweig. The interfacial stability of a ferromagnetic fluid. Journal of Fluid Mechanics, 30(4):671-688, 1967. doi: 10.1017/S0022112067001697.

J. De Vicente, D. J. Klingenberg, and R. Hidalgo-Alvarez. Magnetorheological fluids: a review. Soft matter, 7(8):3701-3710, 2011.

C. Faraci, E. Foti, and R. E. Musumeci. Waves plus currents at a right angle: The rippled bed case. Journal of Geophysical Research: Oceans, 113(C7), 2008. doi: 10.1029/2007JC004468.

K. Y. Lim and O. S. Madsen. An experimental study on near-orthogonal wave-current interaction over smooth and uniform fixed roughness beds. Coastal Engineering, 116:258-274, 2016. ISSN 0378-3839. doi: https://doi.org/10.1016/j.coastaleng.2016.05.005.

C. Lo Re, R. E. Musumeci, and E. Foti. A shoreline boundary condition for a highly nonlinear boussinesq model for breaking waves. Coastal Engineering, 60:41 - 52, 2012. ISSN 0378-3839. doi: https://doi.org/10.1016/j.coastaleng.2011.08.003. URL http: //wWw . sciencedirect.com/science/article/pii/S0378383911001530.

R. E. Musumeci, V. Marletta, B. Andó, S. Baglio, and E. Foti. Ferrofluid measurements of bottom velocities and shear stresses. Journal of Hydrodynamics, Ser. B, 27(1):150-158, 2015a. ISSN 1001-6058. doi: https://doi.org/10.1016/S1001-6058(15)60467-X.

R. E. Musumeci, V. Marletta, B. Andó, S. Baglio, and E. Foti. Measurement of wave near-bed velocity and bottom shear stress by ferrofluids. IEEE Transactions on Instrumentation and Measurement, 64(5): 1224-1231, May 2015b. ISSN 0018-9456. doi: 10.1109/TIM.2014.2359521. 
R. E. Musumeci, V. Marletta, A. Sanchez-Arcilla, and E. Foti. A ferrofluid-based sensor to measure bottom shear stresses under currents and waves. Journal of Hydraulic Research, 56(5):630-647, 2018. doi: 10.1080/00221686.2017.1397779.

S. Odenbach. Ferrofluids: magnetically controllable liquids. PAMM, 1(1):28-32, 2002. doi: 10.1002/ 1617-7061(200203)1:1<28::AID-PAMM28>3.0.CO;2-8.

L. M. Stancanelli, R. E. Musumeci, and E. Foti. Dynamics of gravity currents in the presence of surface waves. Journal of Geophysical Research: Oceans, 123(3):2254-2273, 2018. doi: 10.1002/ $2017 \mathrm{JC} 013273$.

A. Viviano, R. E. Musumeci, and E. Foti. A nonlinear rotational, quasi-2dh, numerical model for spilling wave propagation. Applied Mathematical Modelling, 39(3):1099 - 1118, 2015. ISSN 0307-904X. doi: https://doi.org/10.1016/j.apm.2014.07.030.

J. M. Wallace and P. V. Vukoslavčević. Measurement of the velocity gradient tensor in turbulent flows. Annual Review of Fluid Mechanics, 42:157-181, 2010. 